\title{
On cubic bridgeless graphs whose edge-set cannot be covered by four perfect matchings
}

\author{
L. Esperet ${ }^{*} \quad$ G. Mazzuoccolo ${ }^{\dagger}$
}

October 31, 2018

\begin{abstract}
The problem of establishing the number of perfect matchings necessary to cover the edge-set of a cubic bridgeless graph is strictly related to a famous conjecture of Berge and Fulkerson. In this paper we prove that deciding whether this number is at most 4 for a given cubic bridgeless graph is NP-complete. We also construct an infinite family $\mathcal{F}$ of snarks (cyclically 4-edge-connected cubic graphs of girth at least five and chromatic index four) whose edge-set cannot be covered by 4 perfect matchings. Only two such graphs were known.

It turns out that the family $\mathcal{F}$ also has interesting properties with respect to the shortest cycle cover problem. The shortest cycle cover of any cubic bridgeless graph with $m$ edges has length at least $\frac{4}{3} m$, and we show that this inequality is strict for graphs of $\mathcal{F}$. We also construct the first known snark with no cycle cover of length less than $\frac{4}{3} m+2$.
\end{abstract}

Keywords: Berge-Fulkerson conjecture, perfect matchings, shortest cycle cover, cubic graphs, snarks.

$M S C(2010): 05 C 15$ (05C70)

*Laboratoire G-SCOP (Grenoble-INP, CNRS), Grenoble, France. Partially supported by the French Agence Nationale de la Recherche under reference ANR-10-JCJC-0204-01.

†Laboratoire G-SCOP (Grenoble-INP, CNRS), Grenoble, France. Research supported by a fellowship from the European Project "INdAM fellowships in mathematics and/or applications for experienced researchers cofunded by Marie Curie actions" e-mail: mazzuoccolo@unimore.it 


\section{Introduction}

Throughout this paper, a graph $G$ always means a simple connected finite graph (without loops and parallel edges). A perfect matching of $G$ is a 1regular spanning subgraph of $G$. In this context, a cover of $G$ is a set of perfect matchings of $G$ such that each edge of $G$ belongs to at least one of the perfect matchings. Following the terminology introduced in [3, the excessive index of $G$, denoted by $\chi_{e}^{\prime}(G)$, is the least integer $k$ such that the edge-set of $G$ can be covered by $k$ perfect matchings.

A famous conjecture of Berge and Fulkerson [7] states that the edge-set of every cubic bridgeless graph can be covered by 6 perfect matchings, such that each edge is covered precisely twice. The second author recently proved that this conjecture is equivalent to another conjecture of Berge stating that every cubic bridgeless graph has excessive index at most five [14].

Note that a cubic bridgeless graph has excessive index 3 if and only if it is 3-edge-colorable, and deciding the latter is NP-complete. Hägglund [8, Problem 3] asked if it is possible to give a characterization of all cubic graphs with excessive index 5. In Section 2, we prove that the structure of cubic bridgeless graphs with excessive index at least five is far from trivial. More precisely, we show that deciding whether a cubic bridgeless graph has excessive index at most four (resp. equal to four) is NP-complete.

The gadgets used in the proof of NP-completeness have many 2-edge-cuts, so our first result does not say much about 3-edge-connected cubic graphs. A snark is a non 3-edge-colorable cubic graph with girth (length of a shortest cycle) at least five that is cyclically 4-edge connected. A question raised by Fouquet and Vanherpe [6] is whether the Petersen graph is the unique snark with excessive index at least five. This question was answered by the negative by Hägglund using a computer program [8]. He proved that the smallest snark distinct from the Petersen graph having excessive index at least five is a graph $\stackrel{\circ}{H}$ on 34 vertices (see Figure 7). In Section 3 we show that the graph found by Hägglund is a special member of an infinite family $\mathcal{F}$ of snarks with excessive index precisely five.

In Section 4, we study shortest cycle covers of the graphs from our family $\mathcal{F}$. A cycle cover of a graph $G$ is a covering of the edge-set of $G$ by cycles (connected subgraphs with all degrees even), such that each edge is in at least one cycle. The length of a cycle cover is the sum of the number of edges

in each cycle. The Shortest Cycle Cover Conjecture of Alon and Tarsi [1] 
states that every bridgeless graph $G$ has a cycle cover of length at most $\frac{7}{5}|E(G)|$. This conjecture implies a famous conjecture due to Seymour [15] and Szekeres [17], the Cycle Double Cover Conjecture, which asserts that every bridgeless graph has a cycle cover such that every edge is covered precisely twice (see [12]). It turns out that the Cycle Double Cover Conjecture also has interesting connections with the excessive index of snarks. Indeed, it was proved independently by Steffen [16] and Hou, Lai, and Zhang [10] that it is enough to prove the Cycle Double Cover conjecture for snarks with excessive index at least five.

The best known upper bound on the length of a cycle cover of a bridgeless graph $G, \frac{5}{3}|E(G)|$, was obtained by Alon and Tarsi [1] and Bermond, Jackson, and Jaeger [2]. For cubic bridgeless graphs there is a trivial lower bound of $\frac{4}{3}|E(G)|$, which is tight for 3-edge-colorable cubic graphs. Jackson [11] proved an upper bound of $\frac{64}{39}|E(G)|$, and Fan [5] improved it to $\frac{44}{27}|E(G)|$. The best known upper bound in the cubic case, $\frac{34}{21}|E(G)|$, was obtained by Kaiser, Král', Lidický, Nejedlý, and Š́mal [13] in 2010.

Brinkmann, Goedgebeur, Hägglund, and Markström [4] proved using a computer program that the only snarks $G$ on $m$ edges and at most 36 vertices having no cycle cover of length $\frac{4}{3} m$ are the Petersen graph and the 34 -vertex graph $\stackrel{\circ}{H}$ mentioned above. Moreover, these two graphs have a cycle cover of length $\frac{4}{3} m+1$. They also conjectured that every snark $G$ has a cycle cover of size at most $\left(\frac{4}{3}+o(1)\right)|E(G)|$.

In Section 4, we show that all the graphs $G$ in our infinite family $\mathcal{F}$ have shortest cycle cover of length more than $\frac{4}{3}|E(G)|$. We also find the first known snark with no cycle cover of length less than $\frac{4}{3}|E(G)|+2$ (it has 106 vertices).

Notation Let $X, Y$ be subsets of the vertex-set of $G$. We denote by $\partial X$ the set of edges with precisely one end-vertex in $X$ and by $\partial(X, Y)$ the set of edges with one end-vertex in $X$ and the other in $Y$. If $X, Y$ form a partition of the vertex-set of $G$, the set $\partial(X, Y)$ is called an edge-cut, or a $k$-edge-cut if $k$ is the cardinality of $\partial(X, Y)$. It is well-known that for any $k$-edge-cut $\partial(X, Y)$ and any perfect matching $M$ of a cubic graph $G$, the numbers $|X|$, $|Y|, k$, and $|M \cap \partial(X, Y)|$ have the same parity. This will be used implicitly several times in the remaining of the paper. 


\section{$2 \quad$ NP-completeness}

We denote by $T$ the Tietze graph, that is the graph obtained from the Petersen graph by replacing one vertex by a triangle (see Figure 1).
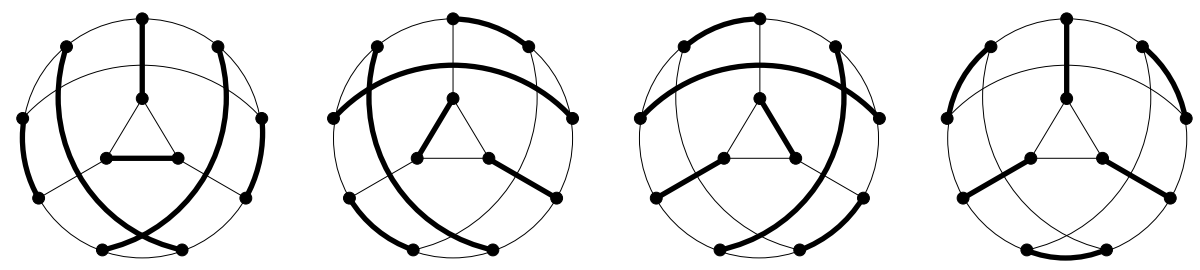

Figure 1: Four perfect matchings covering the edge-set of the Tietze graph.

It is not difficult to check that the Petersen graph has excessive index 5 (see also [6]), while the Tietze graph has excessive index 4 (see Figure 1 for a cover consisting of four perfect matchings). The next lemma describes how four perfect matchings covering the edge-set of $T$ intersect the unique triangle of $T$.

Lemma 1. For any cover $\mathcal{M}$ of $T$ with $|\mathcal{M}|=4$, each edge of the unique triangle of $T$ is in precisely one perfect matching of $\mathcal{M}$.

Proof. By parity, each perfect matching of $\mathcal{M}$ contains one or three edges incident to the unique triangle of $T$. If a perfect matching of $\mathcal{M}$ contains the three edges incident to $T$, then each of the three other perfect matchings of $\mathcal{M}$ must contain a distinct edge of $T$, hence the lemma holds. On the other hand, suppose for the sake of contradiction that each perfect matching of $\mathcal{M}$ contains precisely one edge incident to the triangle. Then by contracting the triangle we obtain four perfect matchings covering the Petersen graph, a contradiction.

Before we prove the main result of this section, we need to describe an operation on cubic graphs. Given two cubic graphs $G$ and $H$ and two edges $x y$ in $G$ and $u v$ in $H$, the gluing 1 of $(G, x, y)$ and $(H, u, v)$ is the graph obtained from $G$ and $H$ by removing edges $x y$ and $u v$, and connecting $x$ and $u$ by an edge, and $y$ and $v$ by an edge. Note that if $G$ and $H$ are cubic and bridgeless, the resulting graph is also cubic and bridgeless.

\footnotetext{
${ }^{1}$ This operation is sometimes called the 2-cut-connection.
} 
When the order of each pair $(x, y)$ and $(u, v)$ is not important, we simply say that we glue the edge uv of $H$ on the edge $x y$ of $G$. We obtain either the gluing of $(G, x, y)$ and $(H, u, v)$ or the gluing of $(G, y, x)$ and $(H, u, v)$, but which one does not matter.

We now study the complexity of bounding the excessive index of a cubic bridgeless graph. A cubic bridgeless graph has excessive index three if and only if it is 3-edge-colorable, and determining the latter is a well-known NPcomplete problem (see [9]). We now prove that determining whether the excessive index is at most 4 (or equal to 4 ) is also hard.

Theorem 2. Determining whether a cubic bridgeless graph $G$ satisfies $\chi_{e}^{\prime}(G) \leq$ 4 (resp. $\left.\chi_{e}^{\prime}(G)=4\right)$ is an NP-complete problem.

Proof. The proof proceeds by reduction to the 3-EDGE-COLORABILITY of cubic bridgeless graphs, which is NP-complete [9]. Our problem is certainly in NP, since a cover of $G$ consisting of four perfect matchings gives a certificate that can be checked in polynomial time.

Now, let $G$ be a cubic bridgeless graph, and let $n$ be its number of vertices. Let $G^{\prime}$ be the graph obtained from $G$ by replacing each vertex by a triangle. Note that $G^{\prime}$ is a cubic bridgeless graph with $3 n$ vertices and $n$ (vertexdisjoint) triangles. The $3 n$ edges of $G^{\prime}$ contained in the $n$ triangles are called the new edges, while the other are called the original edges. Let $u v$ be an edge of the unique triangle of the Tietze graph $T$, and let $H$ be the graph obtained from $G^{\prime}$ and $3 n$ copies of $T$ indexed by the $3 n$ new edges of $G^{\prime}$, by gluing each new edge $e$ of $G$ on the edge $u v$ of the copy $T_{e}$ of $T$. Note that $H$ is a cubic bridgeless graph with $39 n$ vertices. We first remark that if $H$ can be covered by $k$ perfect matchings, then $T$ can also be covered by $k$ perfect matchings since for any copy $T_{e}$, any perfect matching of $H$ contains 0 or 2 edges connecting $T_{e}$ to $G$ (and thus corresponds to a perfect matching of $T$ ). It follows that $H$ is not 3-edge-colorable.

We now prove that $G$ is 3-edge-colorable if and only if $\chi_{e}^{\prime}(H)=4$ (which is equivalent to $\chi_{e}^{\prime}(H) \leq 4$ by the previous remark).

Suppose first that $G$ is 3-edge-colorable, and consider three perfect matchings $M_{1}, M_{2}, M_{3}$ covering the edges of $G$. Each perfect matching $M_{i}, i \in$ $\{1,2,3\}$, can be naturally extended to a perfect matching $M_{i}^{\prime}$ of $G^{\prime}$ such that $\left\{M_{1}^{\prime}, M_{2}^{\prime}, M_{3}^{\prime}\right\}$ covers the edges of $G^{\prime}$. These three perfect matchings can be combined with the three leftmost perfect matchings of Figure 1, for each copy $T_{e}$ glued with $G^{\prime}$, to obtain three perfect matchings of $H$ covering all 
the edges of $H$ except three in each copy of $T$. We add a fourth perfect matching of $H$, obtained by combining the perfect matching of $G^{\prime}$ consisting of the original edges of $G$, with the rightmost perfect matching of Figure 1 in each copy of $T$. Since all the edges of $H$ are covered by these four perfect matchings of $H$, we have $\chi_{e}^{\prime}(H) \leq 4$, and since $H$ is not 3-edge-colorable, $\chi_{e}^{\prime}(H)=4$.

Suppose now that $\chi_{e}^{\prime}(H)=4$, and let $M_{1}, M_{2}, M_{3}, M_{4}$ be perfect matchings covering $H$. By Lemma 1, these perfect matchings correspond to four perfect matchings $M_{1}^{\prime}, M_{2}^{\prime}, M_{3}^{\prime}, M_{4}^{\prime}$ of $G^{\prime}$ covering the edges of $G^{\prime}$, and such that each new edge of $G^{\prime}$ is covered once. Since every vertex of $G^{\prime}$ is incident to two new edges and one original edge, it follows that each original edge is covered twice. For all $i \in\{1,2,3\}$, color the original edges with color $i$ if they are covered by $M_{4}^{\prime}$ and $M_{i}^{\prime}$, or by $M_{j}^{\prime}$ and $M_{k}^{\prime}$ with $\{j, k\}=\{1,2,3\} \backslash\{i\}$. Assume that some original edge $x y$ is covered both by $M_{4}^{\prime}$ and $M_{1}^{\prime}$. Then the two new edges incident to $x$ are covered by $M_{2}^{\prime}$ and $M_{3}^{\prime}$, respectively, and the last edge of the new triangle containing $x$ is covered by one of $M_{4}^{\prime}$ and $M_{1}^{\prime}$. It follows that neither of the two edges incident to this triangle and distinct from $x y$ is covered by $M_{4}^{\prime}$ and $M_{1}^{\prime}$, or by $M_{2}^{\prime}$ and $M_{3}^{\prime}$. By symmetry, it follows that each color class corresponds to a perfect matching of $G$, and so $G$ is 3 -edge-colorable.

\section{An infinite family of snarks with excessive index 5}

Recall that a snark is a cubic graph that is cyclically 4-edge-connected, has girth at least five, and is not 3-edge-colorable. In this section we show how to construct a snark $G$ with excessive index at least 5 from three snarks $G_{0}, G_{1}, G_{2}$ each having excessive index at least 5. Taking $G_{0}=G_{1}=G_{2}$ to be the Petersen graph, we obtain the graph $\stackrel{\circ}{H}$ found by Hägglund using a computer program [8], and for which no combinatorial proof showing that its excessive index is 5 has been known up to now. Our proof holds for any graph obtained using this construction, thus we exhibit an infinite family of snarks with excessive index 5. This answers the question of Fouquet and Vanherpe [6] about the existence of snarks distinct from the Petersen graph having excessive index 5 in a very strong sense. 
The windmill construction 22 For $i \in\{0,1,2\}$, consider a snark $G_{i}$ with an edge $x_{i} y_{i}$. Let $x_{i}^{0}$ and $x_{i}^{1}$ (resp. $y_{i}^{0}$ and $y_{i}^{1}$ ) be the neighbors of $x_{i}$ (resp. $\left.y_{i}\right)$ in $G_{i}$. For $i \in\{0,1,2\}$, let $H_{i}$ be the graph obtained from $G_{i}$ by removing vertices $x_{i}$ and $y_{i}$. We construct a new graph $G$ from the disjoint union of $H_{0}, H_{1}, H_{2}$ and a new vertex $u$ as follows. For $i \in\{0,1,2\}$, we introduce a set $A_{i}=\left\{a_{i}, b_{i}, c_{i}\right\}$ of vertices such that $a_{i}$ is adjacent to $x_{i+1}^{0}$ and $y_{i-1}^{0}, b_{i}$ is adjacent to $x_{i+1}^{1}$ and $y_{i-1}^{1}$, and $c_{i}$ is adjacent to $a_{i}, b_{i}$ and $u$ (here and in the following all indices $i$ are taken modulo 3).

The windmill construction is depicted in Figure 2.
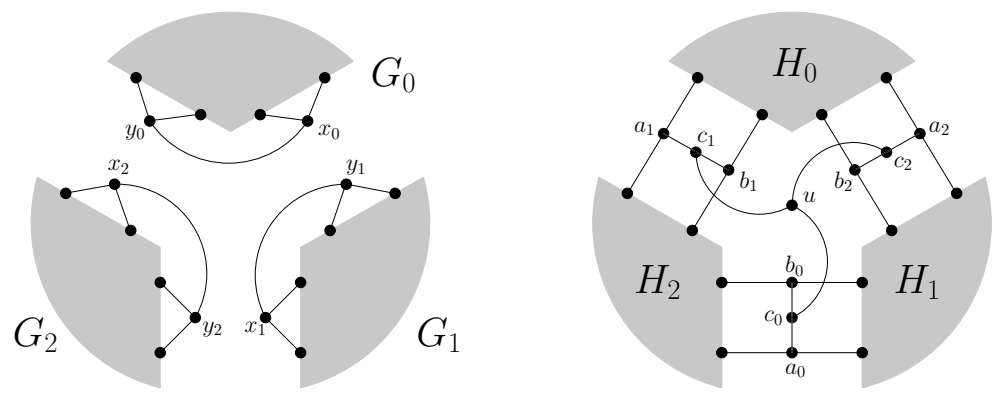

Figure 2: The windmill construction of $G$ (right) from $G_{0}, G_{1}$, and $G_{2}$.

Lemma 3. If $G_{0}, G_{1}, G_{2}$ are snarks, any graph $G$ obtained from $G_{0}, G_{1}, G_{2}$ by the windmill construction is cubic, cyclically 4-edge-connected, and has girth at least 5 .

Proof. It is trivial to check that $G$ is cubic, bridgeless, and has girth at least five (we do not introduce cycles of length three or four, and the distance between any two vertices of any $H_{i}$ is at least as large in $G$ as in $G_{i}$ ). Note that for any $i \in\{0,1,2\}$, the graph $G \backslash\left(V\left(H_{i}\right) \cup V\left(H_{i+1}\right) \cup A_{i-1}\right)$ is a subdivision of $G_{i-1}$. It follows that for any cyclic $k$-edge-cut in $G$, we can find a non-trivial edge-cut of cardinality at most $k$ in some $G_{i}$. If $k \leq 3$ this non-trivial edge-cut is cyclic. Since all $G_{i}$ 's are cyclically 4 -edge-connected, $G$ is also cyclically 4-edge-connected.

Before we prove that $G$ has excessive index at least five, we need a couple of definitions. By a slight abuse of notation, we denote the set of edges of

\footnotetext{
${ }^{2}$ This construction should not be confused with windmill graphs, graphs obtained from disjoint cliques by adding a universal vertex.
} 
$G$ with exactly one end-vertex in $V\left(H_{i}\right)$ by $\partial H_{i}$ (instead of $\partial\left(V\left(H_{i}\right)\right)$ ). In what follows it will be useful to consider how each perfect matching $M$ of $G$ intersects $\partial H_{i}$. Since $\left|\partial H_{i}\right|=4$, by parity we have that $\left|M \cap \partial H_{i}\right|$ is even. Observe that $\left|M \cap \partial H_{i}\right| \neq 4$, since otherwise $u$ would be adjacent to both $c_{i-1}$ and $c_{i+1}$ in $M$. If $\left|M \cap \partial H_{i}\right|=0$, we say that $M$ is of type 0 on $H_{i}$. If $\left|M \cap \partial H_{i}\right|=2$ we consider two cases: we say that $M$ is of type 1 on $H_{i}$ if $\left|M \cap \partial\left(H_{i}, A_{i-1}\right)\right|=\left|M \cap \partial\left(H_{i}, A_{i+1}\right)\right|=1$, while $M$ is of type 2 on $H_{i}$ otherwise (in this case one of the two sets of edges $M \cap \partial\left(H_{i}, A_{i-1}\right)$, $M \cap \partial\left(H_{i}, A_{i+1}\right)$ has cardinality 2 and the other is empty). A perfect matching $M$ of $G$ of type 2 on $H_{0}$, type 1 on $H_{1}$ and type 0 on $H_{2}$ is depicted in Figure 3 (left).
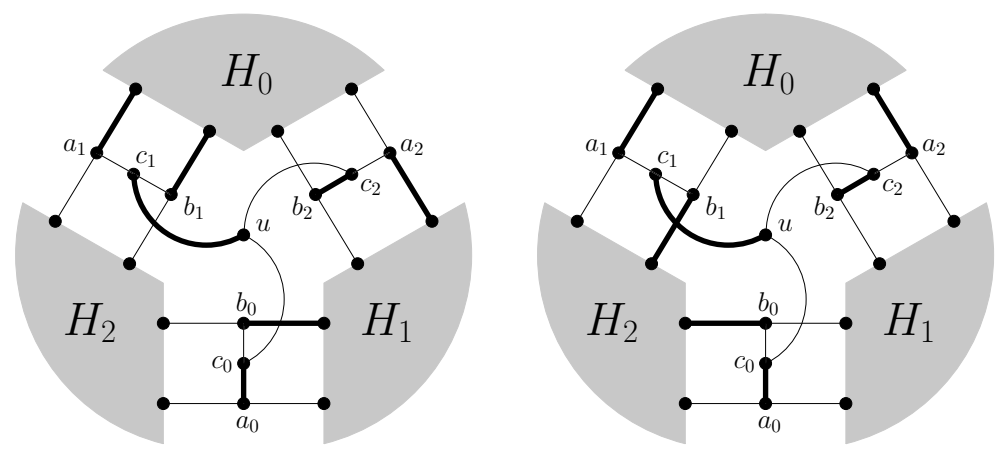

Figure 3: The two kinds of perfect matching of $G$ (up to symmetry).

Observe first that each perfect matching of type 0 on $H_{i}$ corresponds to a perfect matching of $G_{i}$ containing $x_{i} y_{i}$, while each perfect matching of type 1 on $H_{i}$ corresponds to a perfect matching of $G_{i}$ avoiding $x_{i} y_{i}$. This observation has the following immediate consequence:

Lemma 4. Assume that for some $i$ and $k, G$ can be covered by $k$ perfect matchings, each of type 0 or 1 (and not all of type 1) on $H_{i}$. Then $G_{i}$ can be covered by $k$ perfect matchings.

Note that a perfect matching $M$ of $G$ contains two edges of $\partial\left(H_{i-1} \cup\right.$ $\left.H_{i+1}, A_{i}\right)$ if $u c_{i}$ is an edge of $M$, and $M$ contains one edge of $\partial\left(H_{i-1} \cup H_{i+1}, A_{i}\right)$ otherwise. It follows that up to symmetry (including a possible permutation of the $H_{i}$ 's), there are only two kinds of perfect matchings of $G$ : perfect matching of types 0,1 , and 2 (see Figure 3, left), and perfect matching of types 0,1 , and 1 (see Figure 3, right). In particular, we have the following property: 
Observation 5. Every perfect matching of $G$ is of type 2 on at most one $H_{i}$, type 0 on exactly one $H_{i}$, and type 1 on at least one $H_{i}$.

We are now ready to prove our main theorem.

Theorem 6. Let $G_{0}, G_{1}, G_{2}$ be snarks such that $\chi_{e}^{\prime}\left(G_{i}\right) \geq 5$ for $i \in\{0,1,2\}$. Then any graph $G$ obtained from $G_{0}, G_{1}, G_{2}$ by the windmill construction is a snark with $\chi_{e}^{\prime}(G) \geq 5$.

Proof. The graph $G$ is cubic, cyclically 4-edge-connected, and has girth at least 5, as proved in Lemma 3. We now prove that $G$ has excessive index at least five (which implies that $G$ is not 3-edge-colorable, and thus a snark). For the sake of contradiction, let $\mathcal{M}=\left\{M_{1}, M_{2}, M_{3}, M_{4}\right\}$ be a cover of $G$, where $M_{1}$ might be identical to $M_{2}$ (in which case $G$ is 3-edge-colorable).

By Observation 5, each perfect matching $M_{i}$ is of type 0 on one $H_{j}$, so we can assume without loss of generality that $M_{1}$ and $M_{2}$ are of type 0 on $H_{0}$. By Lemma 4, at least one of $M_{3}, M_{4}$ is of type 2 on $H_{0}$, since otherwise $G_{0}$ would have excessive index at most four. If $M_{3}, M_{4}$ do not have the same type on $H_{0}$, then some edge of $\partial H_{0}$ is not covered, so it follows that both $M_{3}, M_{4}$ are of type 2 on $H_{0}$. More precisely, we can assume by symmetry that $\left|\partial\left(H_{0}, A_{1}\right) \cap M_{3}\right|=2$ and $\left|\partial\left(H_{0}, A_{2}\right) \cap M_{4}\right|=2$, i.e. $u c_{1}$ belongs to $M_{3}$ and $u c_{2}$ belongs to $M_{4}$. This implies that $M_{3}$ is of type 1 on $H_{1}$ and of type 0 on $H_{2}$, while $M_{4}$ is of type 0 on $H_{1}$ and of type 1 on $H_{2}$.

Since the edge $u c_{0}$ is not covered by $M_{3} \cup M_{4}$, we can assume without loss of generality that $M_{1}$ contains $u c_{0}$. This implies that $M_{1}$ is of type 1 on both $H_{1}$ and $H_{2}$. Combining this with the conclusion of the previous paragraph, it follows by Lemma 4 that $M_{2}$ is of type 2 on $H_{1}$ and $H_{2}$, which contradicts Observation 5 .

Let $\mathcal{F}$ be the family of all graphs inductively defined as being either the Petersen graph, or a graph obtained from three graphs $G_{0}, G_{1}, G_{2}$ of $\mathcal{F}$ by the windmill construction. Theorem 6 shows that $\mathcal{F}$ is an infinite family of snarks with excessive index at least 5 .

We now prove that every graph from $\mathcal{F}$ has excessive index precisely five. We will indeed prove the stronger statement that every graph of $\mathcal{F}$ satisfies the Berge-Fulkerson conjecture, i.e. has six perfect matchings covering each edge precisely twice. Such a cover of a cubic bridgeless graph $G$, called a Berge-Fulkerson cover, can be equivalently defined as a coloring $C$ of the edges of $G$ with subsets of size two of $\{1,2,3,4,5,6\}$ such that for each 
triple of edges $e_{1}, e_{2}, e_{3}$ sharing a vertex, the sets $C\left(e_{i}\right)$ are pairwise disjoint (equivalently, $\left.C\left(e_{1}\right) \cup C\left(e_{2}\right) \cup C\left(e_{3}\right)=\{1,2,3,4,5,6\}\right)$.

Lemma 7. If $G_{0}, G_{1}, G_{2}$ are cubic bridgeless graphs with a Berge-Fulkerson cover, then any graph $G$ obtained from $G_{0}, G_{1}, G_{2}$ by the windmill construction has a Berge-Fulkerson cover.

Proof. Consider a Berge-Fulkerson cover $C$ of $G_{0}$, and let $e_{1}, e_{2}, e_{3}, e_{4}$ be the four edges sharing a vertex with the edge $x_{0} y_{0}$, such that $e_{1}, e_{2}$ are incident to $x_{0}$ and $e_{3}, e_{4}$ are incident to $y_{0}$. Let $\{\alpha, \beta\}=C\left(e_{1}\right)$ and $\{\gamma, \delta\}=C\left(e_{2}\right)$. Then at least one of $\gamma, \delta$, say $\gamma$, is such that $C\left(e_{3}\right)$ and $C\left(e_{4}\right)$ are distinct from $\{\alpha, \gamma\}$. It follows that, after renaming the colors of $C$, we can assume without loss of generality that $i \in C\left(e_{i}\right)$ for all $1 \leq i \leq 4$, while $x_{0} y_{0}$ is colored $\{5,6\}$.
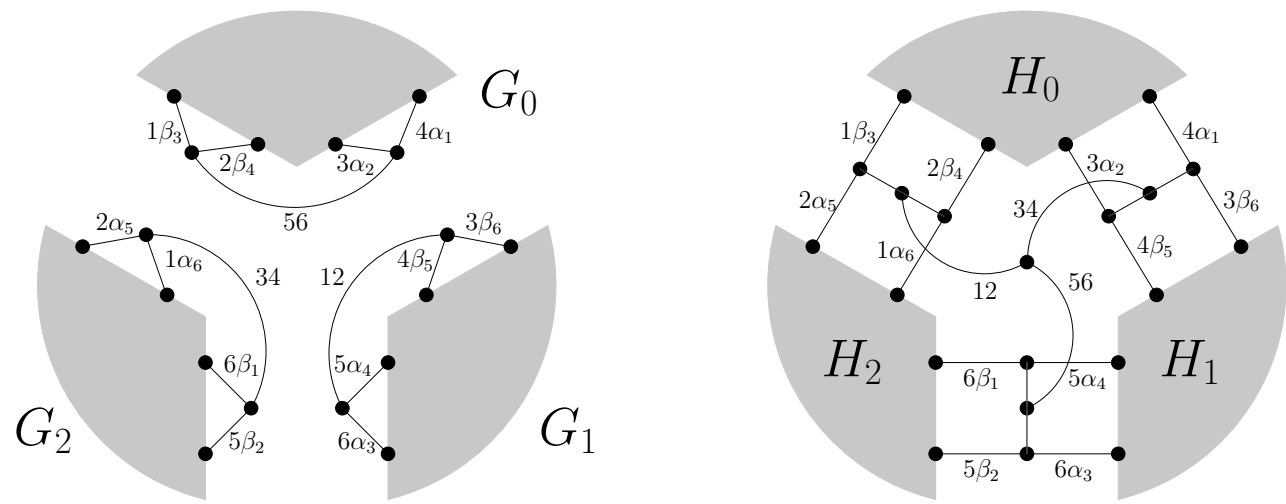

Figure 4: The extension of Berge-Fulkerson covers of $G_{0}, G_{1}, G_{2}$ to $G$. Colors are denoted by $i j$ instead of $\{i, j\}$.

By symmetry, it follows that $G_{0}, G_{1}, G_{2}$ have a Berge-Fulkerson cover as depicted in Figure 4 (left), where for every $(i, j) \in\{(1,2),(3,4),(5,6)\}$, $\left\{\alpha_{i}, \alpha_{j}\right\}=\left\{\beta_{i}, \beta_{j}\right\}=\{i, j\}$. These covers can be extended into a BergeFulkerson cover of $G$, as described in Figure 4 (right). In order not to overload the figure, we have omitted the colors of the edges $a_{i} c_{i}$ and $b_{i} c_{i}, 0 \leq i \leq$ 2. It can be checked that taking $C\left(a_{0} c_{0}\right)=\left\{\beta_{1}, \alpha_{4}\right\}, C\left(b_{0} c_{0}\right)=\left\{\beta_{2}, \alpha_{3}\right\}$, $C\left(a_{1} c_{1}\right)=\left\{\beta_{4}, \alpha_{6}\right\}, C\left(b_{1} c_{1}\right)=\left\{\beta_{3}, \alpha_{5}\right\}, C\left(a_{2} c_{2}\right)=\left\{\alpha_{2}, \beta_{5}\right\}$, and $C\left(b_{2} c_{2}\right)=$ $\left\{\alpha_{1}, \beta_{6}\right\}$ defines a Berge-Fulkerson cover of $G$.

Theorem 6 and Lemma 7 have the following immediate corollary: 
Corollary 8. The family $\mathcal{F}$ is an infinite family of snarks having excessive index precisely five.

\section{Shortest cycle cover}

The length of a shortest cycle cover of a bridgeless graph $G$ is denoted by $\operatorname{scc}(G)$. Note that for any cubic graph $G, \operatorname{scc}(G) \geq \frac{4}{3}|E(G)|$. The purpose of this section is to show that for any graph $G$ in the family $\mathcal{F}$ constructed in the previous section, $\operatorname{scc}(G)>\frac{4}{3}|E(G)|$. Given some integer $k$, a $k$-parity subgraph of a cubic graph $G$ is a spanning subgraph of $G$ in which all vertices have degree one, except $k$ vertices that have degree 3 . We first observe the following:

Observation 9. For any cubic graph $G$ and any cycle cover $\mathcal{C}$ of $G, \mathcal{C}$ has length $\frac{4}{3}|E(G)|$ if and only if the set of edges covered twice by $\mathcal{C}$ is a perfect matching of $G$; and $\mathcal{C}$ has length $\frac{4}{3}|E(G)|+1$ if and only if the set of edges covered twice by $\mathcal{C}$ is a 1-parity subgraph of $G$.

Proof. For each vertex $v$ of $G$, let $d_{\mathcal{C}}(v)$ be the sum of the degree of $v$ in each of the cycles of $\mathcal{C}$. Since $\mathcal{C}$ is a cycle cover and $G$ is cubic, for each vertex $v$, $d_{\mathcal{C}}(v) \geq 3$ and $d_{\mathcal{C}}(v)$ is an even integer. In particular $d_{\mathcal{C}}(v) \geq 4$. It follows that $\mathcal{C}$ has length $\frac{4}{3}|E(G)|$ if and only if for each vertex $v, d_{\mathcal{C}}(v)=4$. This is equivalent to the fact that two edges incident to $v$ are covered once by $\mathcal{C}$, and the third edge is covered twice by $\mathcal{C}$. Hence, $\mathcal{C}$ has length $\frac{4}{3}|E(G)|$ if and only if the set of edges covered twice by $\mathcal{C}$ is a perfect matching of $G$.

Similarly, $\mathcal{C}$ has length $\frac{4}{3}|E(G)|+1$ if and only if there is a vertex $u$ with $d_{\mathcal{C}}(u)=6$ and for each vertex $v \neq u, d_{\mathcal{C}}(v)=4$. Note that as above, if $d_{\mathcal{C}}(v)=4$, then two edges incident to $v$ are covered once by $\mathcal{C}$, and the third edge is covered twice by $\mathcal{C}$. In particular, no edge of $G$ is covered more than twice by $\mathcal{C}$. Since $d_{\mathcal{C}}(u)=6$, the only possibility is that each of the three edges incident to $u$ is covered twice by $\mathcal{C}$. Equivalently, the set of edges covered twice by $\mathcal{C}$ is a 1-parity subgraph of $G$.

Let $G_{0}, G_{1}, G_{2}$ be snarks and let $G$ be a snark obtained from $G_{0}, G_{1}, G_{2}$ by the windmill construction. Recall that the type of a perfect matching of $G$ on $H_{i}$ was defined in the previous section. We define the type of a 1-parity subgraph $P$ of $G$ on $H_{i}$ similarly: If $\left|P \cap \partial H_{i}\right|=j$ with $j \in\{0,4\}$, we say that $P$ is of type $j$ on $H_{i}$ (as it was observed in the previous section, type 4 cannot 
occur when $P$ is a perfect matching). If $\left|P \cap \partial H_{i}\right|=2$ we consider two cases: we say that $P$ is of type 1 on $H_{i}$ if $\left|P \cap \partial\left(H_{i}, A_{i-1}\right)\right|=\left|P \cap \partial\left(H_{i}, A_{i+1}\right)\right|=1$, while $P$ is of type 2 on $H_{i}$ otherwise (in this case one of the two sets of edges $P \cap \partial\left(H_{i}, A_{i-1}\right), P \cap \partial\left(H_{i}, A_{i+1}\right)$ has cardinality 2 and the other is empty).

Let $\mathcal{C}$ be a cycle cover of $G$. For any edge $e$, we denote by $\mathcal{C}(e)$ the set of cycles of $\mathcal{C}$ containing the edge $e$. In the next two lemmas, we fix $i, j \in\{0,1,2\}$ with $i \neq j$, and call $e_{j}$ and $f_{j}$ the edges connecting $H_{i}$ to $a_{j}$ and $b_{j}$ respectively.

Lemma 10. Assume $G$ has a cycle cover $\mathcal{C}$ of length $\frac{4}{3}|E(G)|$. Let $M$ be the perfect matching of $G$ consisting of the edges covered twice by $\mathcal{C}$. If there are distinct cycles $x, y \in \mathcal{C}$, such that either (i) $M$ is of type 0 on $H_{i}$ and $\left\{\mathcal{C}\left(e_{j}\right), \mathcal{C}\left(f_{j}\right)\right\}=\{\{x\},\{y\}\}$, or (ii) $M$ is of type 1 on $H_{i}$ and $\left\{\mathcal{C}\left(e_{j}\right), \mathcal{C}\left(f_{j}\right)\right\}=$ $\{\{x\},\{x, y\}\}$, then $G_{i}$ has a cycle cover $\mathcal{C}_{i}$ of length $\frac{4}{3}\left|E\left(G_{i}\right)\right|$.

Proof. In both cases we obtain a cycle cover of $G_{i}$ such that the set of edges covered twice is a perfect matching of $G_{i}$ (see Figure 5, where cycles of $G_{i}$ are represented by dashed or dotted lines for more clarity). By Observation 9 this cycle cover has length $\frac{4}{3}\left|E\left(G_{i}\right)\right|$. Note that in case (ii) (see Figure 5, right), $x$ and $z$ might be the same cycle.
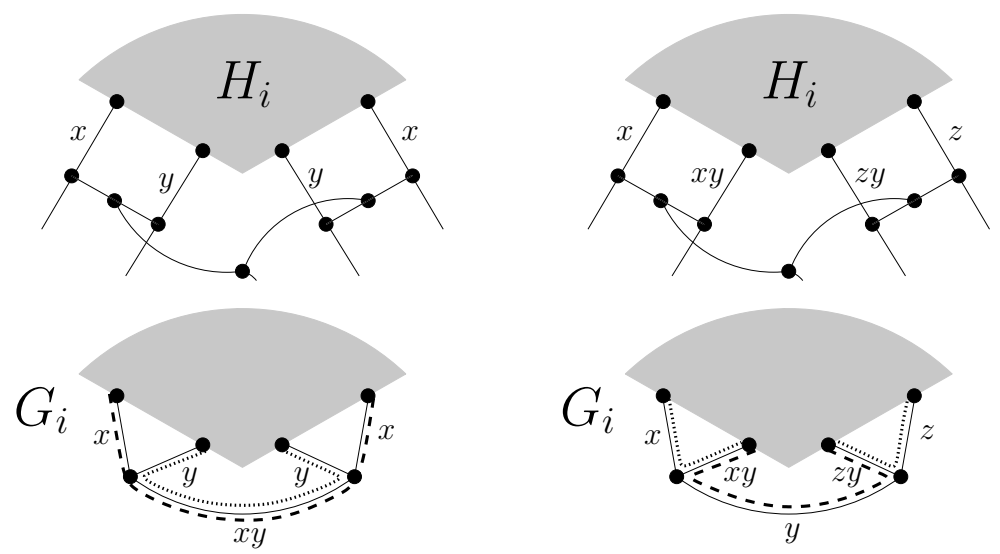

Figure 5: Constructing cycle covers of $G_{i}$ from cycle covers of $G$.

Given a cycle cover $\mathcal{C}$ of $G$ of length $\frac{4}{3}|E(G)|+1$, the unique vertex of degree 3 in the 1-parity subgraph of $G$ associated to $\mathcal{C}$ will be denoted by $t_{\mathcal{C}}$ (or simply $t$ if the cycle cover is implicit). The same proof as above, applied this time to 1-parity subgraphs, gives the following slightly different lemma. 
Lemma 11. Assume $G$ has a cycle cover $\mathcal{C}$ of length $\frac{4}{3}|E(G)|+1$. Let $P$ be the 1-parity subgraph of $G$ consisting of the edges covered twice by $\mathcal{C}$. If there are distinct cycles $x, y \in \mathcal{C}$, such that either (i) $P$ is of type 0 on $H_{i}$ and $\left\{\mathcal{C}\left(e_{j}\right), \mathcal{C}\left(f_{j}\right)\right\}=\{\{x\},\{y\}\}$, or (ii) $P$ is of type 1 on $H_{i}$ and $\left\{\mathcal{C}\left(e_{j}\right), \mathcal{C}\left(f_{j}\right)\right\}=$ $\{\{x\},\{x, y\}\}$, then $G_{i}$ has a cycle cover $\mathcal{C}_{i}$ of length at most $\frac{4}{3}\left|E\left(G_{i}\right)\right|+1$. Moreover, if $t_{\mathcal{C}}$ does not lie in $H_{i}$ or its neighborhood in $G$, then $\mathcal{C}_{i}$ has length at most $\frac{4}{3}\left|E\left(G_{i}\right)\right|$.

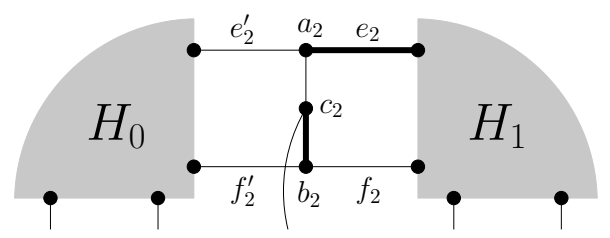

Figure 6: Notation in the proof of Theorem [12,

We now use Lemma 10 to prove the main result of this section.

Theorem 12. Let $G_{0}, G_{1}, G_{2}$ be snarks such that $\operatorname{scc}\left(G_{i}\right)>\frac{4}{3}\left|E\left(G_{i}\right)\right|$ for $i \in\{0,1,2\}$. Then any graph $G$ obtained from $G_{0}, G_{1}, G_{2}$ by the windmill construction is a snark with $\operatorname{scc}(G)>\frac{4}{3}|E(G)|$.

Proof. Assume that $G$ has a cycle cover $\mathcal{C}$ of length $\frac{4}{3}|E(G)|$, and let $M$ be the perfect matching consisting of the edges covered twice by $\mathcal{C}$. By Observation 5. we can assume that $M$ is of type 0 on $H_{0}$ and type 1 on $H_{1}$. Let $e_{2}, f_{2}$ be the edges connecting $H_{1}$ to $a_{2}, b_{2}$ and let $e_{2}^{\prime}, f_{2}^{\prime}$ be the edges connecting $H_{0}$ to $a_{2}, b_{2}$ (see Figure 6). By symmetry we can assume that $e_{2}$ and $b_{2} c_{2}$ are in $M$. Let $x, y \in \mathcal{C}$ be the two cycles covering $e_{2}$. Then one of them, say $x$, also covers $b_{2} c_{2}$, and therefore covers either $f_{2}$ or $f_{2}^{\prime}$. If $x$ covers $f_{2}^{\prime}$ we obtain a contradiction with Lemma 10, case (i), and if $x$ covers $f_{2}$ we obtain a contradiction with Lemma 10, case (ii).

Recall that beside the Petersen graph, only one snark $G$ with $\operatorname{scc}(G)>$ $\frac{4}{3}|E(G)|$ was known [4] (and the proof of it was computer-assisted). Theorem 12 has the following immediate corollary:

Corollary 13. The family $\mathcal{F}$ is an infinite family of snarks such that for any $G \in \mathcal{F}, \operatorname{scc}(G)>\frac{4}{3}|E(G)|$. 


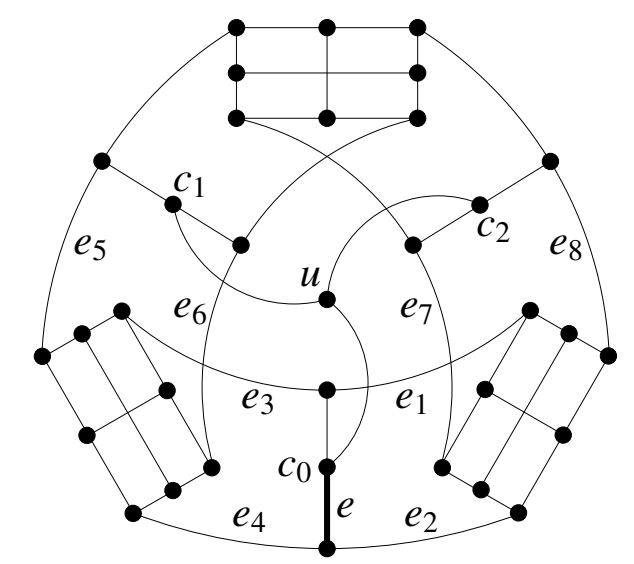

Figure 7: The graph $\stackrel{\circ}{H}$ in Lemma 14. The bold edge $e$ will be used in the proof of Theorem [15.

We now prove that in a cycle cover $\mathcal{C}$ of the graph $\stackrel{\circ}{H}$ of Figure 7 of length $\frac{4}{3}|E(\stackrel{\circ}{H})|+1$, some vertices cannot play the role of $t_{\mathcal{C}}$ (the only vertex of degree 3 in the 1-parity subgraph of $\stackrel{\circ}{H}$ associated to $\mathcal{C})$.

Lemma 14. Let $G_{0}, G_{1}, G_{2}$ be three copies of the Petersen graph. Then the graph $\stackrel{\circ}{H}$ obtained from $G_{0}, G_{1}, G_{2}$ by the windmill construction is a snark such that:

1. $\operatorname{scc}(\stackrel{\circ}{H})=\frac{4}{3}|E(\stackrel{\circ}{H})|+1$

2. For every cycle cover $\mathcal{C}$ of $\stackrel{\circ}{H}$ of minimum length, the vertex $t_{\mathcal{C}}$ cannot be one of the vertices $c_{i}$ (see Figure 7).

Proof. The first part of the lemma was proved in [4]. Assume now that the second part fails for some cycle cover $\mathcal{C}$ of length $\frac{4}{3}|E(\stackrel{\circ}{H})|+1$, and let $P$ be the 1-parity subgraph associated to $\mathcal{C}$. By symmetry, we suppose $t_{\mathcal{C}}=c_{0}$. It implies that all three edges incident to $c_{0}$ are in $P$. As a consequence, each edge $e_{i}, 1 \leq i \leq 4$, is covered only once by $\mathcal{C}$ (see Figure 7 ). It follows also that one edge incident to $c_{1}$ and distinct from $c_{1} u$ is covered twice, and by parity this implies that $e_{5}$ and $e_{6}$ are covered once. By a symmetric argument, $e_{7}$ and $e_{8}$ are covered once, therefore $P$ is of type 0 on $H_{1}$ and $H_{2}$. The edges incident to $c_{0}$ are covered by three different cycles, so at least one of the pairs $e_{1}, e_{2}$ and $e_{3}, e_{4}$ is such that the two edges in the pair are not covered by the same cycle of $\mathcal{C}$. This contradicts Lemma 11, case (i), since the Petersen graph has no cycle cover of length 20. 


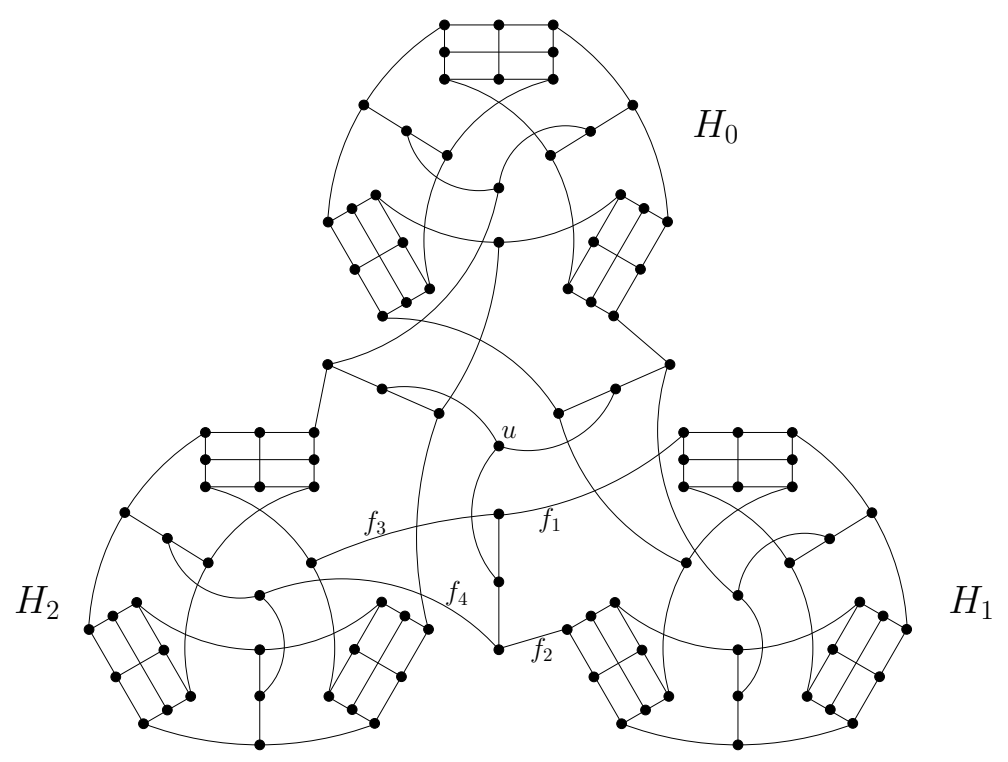

Figure 8: A snark $G$ with $\operatorname{scc}(G) \geq \frac{4}{3}|E(G)|+2$.

We now use Lemma 14 to exhibit the first known snark $G$ satisfying $\operatorname{scc}(G)>\frac{4}{3}|E(G)|+1$.

Theorem 15. Any cycle cover of the graph $G$ depicted in Figure 8 has length at least $\frac{4}{3}|E(G)|+2$.

Proof. The graph $G$ is obtained from three copies $G_{0}, G_{1}, G_{2}$ of the graph $\stackrel{\circ}{H}$ of Figure 7 by the windmill construction, where each $H_{i}$ is obtained from $G_{i}$ by removing the endpoints of the edge $e$ (see Figure 7). By Theorem 12, $\operatorname{scc}(G)>\frac{4}{3}|E(G)|$. Assume for the sake of contradiction that $G$ has a cycle cover $\mathcal{C}$ of length $\frac{4}{3}|E(G)|+1$, and let $P$ be the 1-parity subgraph associated to $\mathcal{C}$ and $t=t_{\mathcal{C}}$ be the only vertex of degree three in $P$.

We claim that if $P$ is of type 0 or 1 on $H_{i}$, then $t$ lies in $H_{i}$. For the sake of contradiction, assume that $P$ is of type 0 or 1 on $H_{i}$, while $t$ lies outside of $H_{i}$ (note that $t$ might be adjacent to some vertex of $H_{i}$ ). Then $P$ induces a perfect matching of $G_{i}$. The graph $G_{i}$ itself was obtained from three copies $G_{0}^{\prime}, G_{1}^{\prime}, G_{2}^{\prime}$ of the Petersen graph by the windmill construction. By Observation 5, $P$ is of type 0 on some $G_{i}^{\prime}$, and type 1 on some $G_{j}^{\prime}$. Therefore we can assume, following the notation of Figure 7 , that $e, e_{1} \in P$ and $e_{2}, e_{3}, e_{4} \notin P$. Since the Petersen graph has no cycle cover of length 20, we can assume by Lemma 10, case (i), that $e_{3}$ and $e_{4}$ belong to the same 
cycle of $\mathcal{C}$, and by Lemma 10, case (ii), that the cycle containing $e_{2}$ does not contain $e_{1}$. It can be checked that this is a contradiction.

Now, if $t$ lies inside some $H_{i}$, remark that $P$ satisfies the conclusion of Observation 5 (since the proof of this observation only considers the intersection of $P$ and the edges outside the $H_{i}$ 's). In particular, $P$ is of type 0 on some $H_{j}$, and type 1 on some $H_{k}$. This contradicts the claim of the previous paragraph. Similarly, if $t$ is outside of the $H_{i}$ 's, but distinct from $u$ (see Figure 8), it can be checked that $P$ is of type 1 on some $H_{i}$, which again contradicts the previous paragraph. It follows that $t$ coincides with $u$ and $P$ is of type 2 or 4 on each $H_{i}$. This implies that $P$ is of type 2 on each $H_{i}$, so either the two edges $f_{1}, f_{2}$ or the two edges $f_{3}, f_{4}$ belong to $P$. Then $P$ induces a 1-parity subgraph of $G_{1}$ (in the first case) or $G_{2}$ (in the second case), such that the unique vertex of degree three of this 1-parity subgraph coincides with a vertex $c_{j}$ of $G_{i}$, contradicting Lemma 14,

\section{Open problems}

Hägglund proposed the following two problems (Problems 3 and 4 in [8]):

1. Is it possible to give a simple characterization of cubic graphs $G$ with $\chi_{e}^{\prime}(G)=5 ?$

2. Are there any cyclically 5 -edge-connected snarks $G$ with excessive index at least five distinct from the Petersen graph?

While the former problem has a negative answer by Theorem 2 of the present paper (unless $P=N P$ ), the latter one is still open, since each element of the infinite family $\mathcal{F}$ contains cyclic 4 -edge-cuts. The edge-connectivity also plays an important role in the proof of Theorem 2, in particular the gadgets we use have many 2-edge-cuts. Hence we leave open the problem of establishing whether it is possible to give a simple characterization of 3-edge-connected or cyclically 4-edge-connected cubic graphs with excessive index 5 .

In Section 4, we have shown interesting properties of $\mathcal{F}$ with respect to cycle covers. In particular, Theorem 15 proves the existence of a snark $G \in \mathcal{F}$ with no cycle cover of length less than $\frac{4}{3}|E(G)|+2$. We believe that there exist snarks in $\mathcal{F}$ for which the constant 2 can be replaced by an arbitrarily large number. On the other hand, recall that Brinkmann, Goedgebeur, Hägglund, and Markström [4] conjectured that every snark $G$ has a cycle cover of size at most $\left(\frac{4}{3}+o(1)\right)|E(G)|$. 


\section{References}

[1] N. Alon and M. Tarsi, Covering multigraphs by simple circuits, SIAM J. Algebraic Discrete Methods 6 (1985), 345-350.

[2] J.C. Bermond, B. Jackson, and F. Jaeger, Shortest coverings of graphs with cycles, J. Combin. Theory Ser. B 35 (1983), 297-308.

[3] A. Bonisoli and D. Cariolaro, Excessive Factorizations of Regular Graphs, in: A. Bondy et al. (Eds.), Graph theory in Paris, Birkhäuser, Basel, (2007), 73-84.

[4] G. Brinkmann, J. Goedgebeur, J. Hägglund, and K. Markström, Generation and properties of snarks, J. Combin. Theory Ser. B, 103 (2013) 468-488.

[5] G. Fan, Shortest cycle covers of cubic graphs, J. Graph Theory 18 (1994), 131-141.

[6] J.L. Fouquet and J.M. Vanherpe, On the perfect matching index of bridgeless cubic graphs, Manuscript, 2009. arXiv:0904.1296.

[7] D.R. Fulkerson, Blocking and anti-blocking pairs of polyhedra, Math. Programming 1 (1971), 168-194.

[8] J. Hägglund, On snarks that are far from being 3-edge colorable, Manuscript, 2012. arXiv:1203.2015.

[9] I. Holyer, The NP-completeness of edge-coloring, SIAM J. Comput. 10 (1981), 718-720.

[10] X. Hou, H.J. Lai, and C.Q. Zhang, On matching coverings and cycle coverings, Manuscript, 2012.

[11] B. Jackson, Shortest circuit covers of cubic graphs, J. Combin. Theory Ser. B 60 (1994), 299-307.

[12] U. Jamshy and M. Tarsi, Shortest cycle covers and the cycle double cover conjecture, J. Combin. Theory Ser. B 56 (1992), 197-204. 
[13] T. Kaiser, D. Král', B. Lidický, P. Nejedlý, and R. Šámal, Short cycle covers of cubic graphs and graphs with minimum degree three, SIAM J. Discrete Math. 24 (2010), 330-355.

[14] G. Mazzuoccolo, The equivalence of two conjectures of Berge and Fulkerson, J. Graph Theory 68 (2011) 125-128.

[15] P.D. Seymour, Sums of circuits, in: Graph theory and related topics (J. A. Bondy and U. S. R. Murty, eds.), Academic Press, New York (1979), 342-355.

[16] E. Steffen, 1-factor and cycle covers of cubic graphs, Manuscript, 2012. arXiv:1209.4510.

[17] G. Szekeres, Polyhedral decompositions of cubic graphs, Bull. Austral. Math. Soc. 8 (1973), 367-387. 